\title{
11. Circulación pulmonar y trombosis
}

\subsection{Accesos vasculares y desenlaces en fibrinolisis: registro local y recomendaciones \\ Radillo Alba Hugo Alberto \\ Hospital Civil de Guadalajara Fray Antonio Alcalde. \\ Tipo de estudio: Registros locales o nacionales}

Introducción: La fibrinolisis es una estrategia contemporánea para limitar la pérdida de la función de tejidos, cuyo flujo sanguíneo se ve comprometido por un coágulo de fibrina y componentes hemáticos. Desafortunadamente, una fracción de los pacientes candidatos a fibrinolisis están en un contexto crítico, meritorio de invasión vascular con catéteres de calibre considerable y con riesgo inherente de hemorragias localizadas. Objetivo: Documentar evidencia sobre la relación con los desenlaces al seleccionar una vía central o periférica, para administrar fibrinolíticos en el contexto de cardiopatía isquémica y tromboembolismo pulmonar. Metodología: En 36 meses se realizó un registro local con seguimiento intrahospitalario en pacientes con infarto miocárdico y elevación del segmento ST y tromboembolismo pulmonar de riesgo elevado, meritorios de fibrinolisis. Se enrolaron 22 pacientes cuya terapia con fibrinolítico se administró por catéter periférico, 22 por catéter yugular y 22 por catéter subclavio. Se excluyeron del registro pacientes con discrasias sanguíneas. Resultados: La fibrinolisis fue exitosa en más del $80 \%$ de los pacientes involucrados. Dos pacientes manifestaron hemorragia significativa (un paciente en el grupo de catéter periférico y uno en el grupo de catéter yugular). El 13.6\% de los pacientes en el grupo de catéter yugular presentó hematomas en el sitio de punción. La estancia hospitalaria en pacientes con catéter yugular fue 6.4 días y 3.1 en el grupo de accesos periféricos. Discusión y conclusiones: No se observó diferencia entre las vías de administración y la eficacia del fibrinolítico o los riesgos hemorrágicos. La estancia hospitalaria fue mayor en pacientes con accesos yugulares.

\subsection{Evento vascular cerebral tromboembólico como manifestación de malformación arteriovenosa pulmonar}

Blanco Velasco Daniel Gilberto, Márquez-González Horacio, Yáñez-Gutiérrez Lucelli, Salazar-Lizárraga David, García-Dávalos Israel, López-Gallegos Diana, Germán-Arroyo Cristopher Instituto Mexicano del Seguro Social.

Tipo de estudio: Reporte de casos clínicos con revisión de fuentes

Introducción: Las malformaciones arteriovenosas pulmonares (MAVP) son estructuras vasculares que consisten en la conexión entre una arteria y vena pulmonares causando un shunt de derecha a izquierda dentro de los pulmones. Descripción del caso: Hombre de 26 años con evento vascular cerebral (EVC) embólico secundario a probable CIA. Inició padecimiento con acropaquias, acrocianosis, disnea y deterioro clase funcional, saturación inicial $82 \%$. Se realizó TAC observando trayectos arteriales y venosos con dilatación y fístula arteriovenosa pulmonar en la rama del lóbulo posterosuperior. Se realizó cateterismo terapéutico y se colocó dispositivo Amplatzer vascular Plug II. Metodología: Mediante el motor de búsqueda PubMed se identificaron artículos de revisión y reportes de caso que cumplieran con los criterios de aplicabilidad, reproducibilidad y similitud a las características de nuestro paciente, encontrándose múltiples reportes de caso con abordaje diagnóstico y tratamiento similar a nuestro caso. Resultados: Más de la mitad de los pacientes con MAVP cursan asintomáticos, cerca del 40\% tienen manifestaciones neurológicas como son EVC y crisis convulsivas, y sólo el 10\% presentan disnea y cianosis. Por lo que al presentarse un paciente de edad joven con cianosis y eventos embólicos, es indispensable realizar un estudio anatómico completo. Discusión: Las MAVP son alteraciones vasculares que cuentan con una elevada morbilidad y mortalidad si no se tratan, actualmente los estudios de imagen, como son rayos $\mathrm{X}$ y tomografía computarizada, nos permiten realizar un

Tabla 11.1.1: Características de los pacientes asociadas a desenlaces.

\begin{tabular}{|c|c|c|c|}
\hline & \multicolumn{3}{|c|}{ Acceso vascular } \\
\hline & $\begin{array}{c}\text { Subclavio }(\mathrm{n}=22) \\
\mathrm{n}(\%)\end{array}$ & $\begin{array}{c}\text { Yugular }(\mathrm{n}=22) \\
\mathrm{n}(\%)\end{array}$ & $\begin{array}{c}\text { Periférico }(\mathrm{n}=22) \\
\mathrm{n}(\%)\end{array}$ \\
\hline Fibrinolisis exitosa & $18(82)$ & $20(90)$ & $19(86)$ \\
\hline Hemorragia grave & $0 \%$ & $1(4.5)$ & $1(4.5)$ \\
\hline Hematoma en sitio de punción & $1(4.5)$ & $3(13.6)$ & $0 \%$ \\
\hline Proceso infeccioso subyacente & $2(9)$ & $2(9)$ & $1(4.5)$ \\
\hline Anticoagulación previa & $17(77.2)$ & $20(90)$ & $18(82)$ \\
\hline Doble antiagregación previa & $3(13.6)$ & $1(4.5)$ & $3(13.6)$ \\
\hline Tensión arterial sistólica > 140 mmHg* & $0 \%$ & $0 \%$ & $1(4.5)$ \\
\hline Tensión arterial diastólica > 90 mmHg* & $0 \%$ & $0 \%$ & $1(4.5)$ \\
\hline Mortalidad & $1(4.5)$ & $1(4.5)$ & $2(9)$ \\
\hline Días de estancia hospitalaria promedio & $2-8(4.4)$ & $2-10(6.4)$ & $1-5(3.1)$ \\
\hline
\end{tabular}

* Tensión arterial tomada previo a fibrinólisis. 
diagnóstico preciso de esta patología. El abordaje mediante radiología intervencionista es actualmente la primera línea de tratamiento con prácticamente una muy baja morbilidad y mortalidad.

\subsection{Seguridad y efectividad de media dosis de alteplasa en tromboembolia pulmonar masiva y submasiva}

Paredes Vázquez José Gildardo, Jerjes-Sánchez Carlos,

Castro-Varela Alejandra, Rodríguez David

Tecnológico de Monterrey.

Tipo de estudio: Reporte de casos clínicos con revisión de fuentes

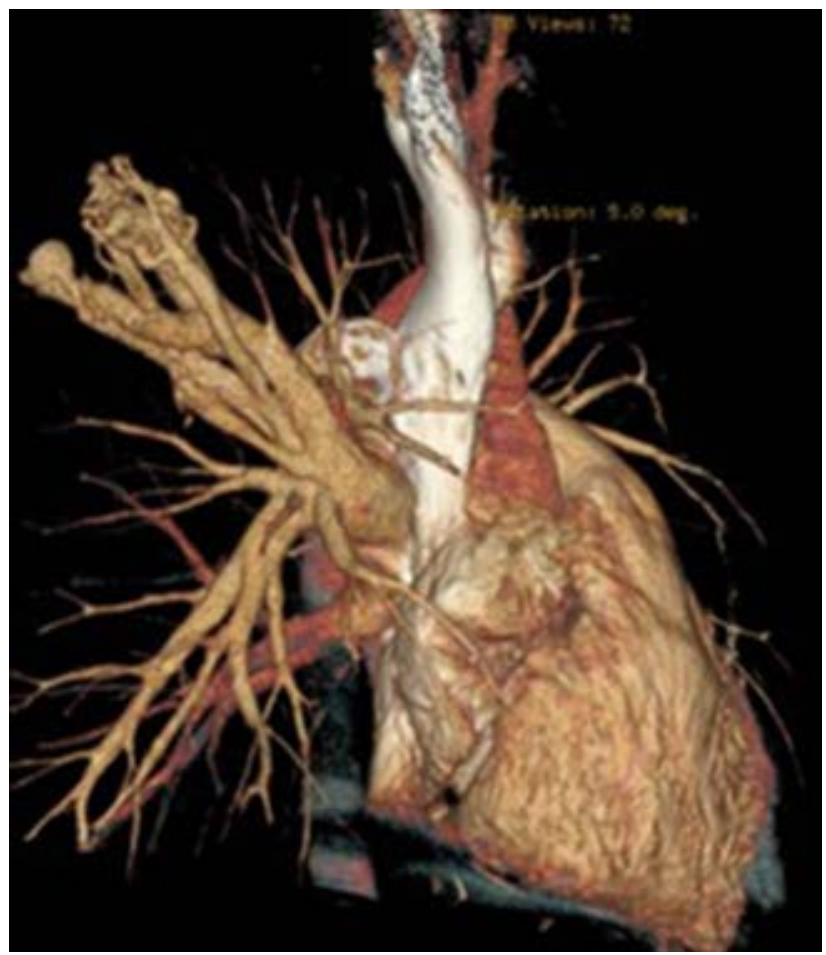

Figura 11.2.1.
Introducción: Alteplasa $100 \mathrm{mg}$ en tromboembolia pulmonar (TEP) submasiva demostró disminuir mortalidad y el deterioro clínico, a expensas de una mayor incidencia de complicaciones hemorrágicas. Evidencias recientes sugieren que dosis reducidas tienen igual efectividad y mayor seguridad. Descripción del caso: Analizar la seguridad y efectividad de $50 \mathrm{mg}$ de alteplasa en TEP submasiva y masiva confirmada comparada con 100 mg y sólo heparina. Metodología: Revisión sistematizada y metaanálisis. Base de datos electrónicas. PRISMA y PROSPERO. Términos MeSH. Estudios controlados, observacionales, cohortes, series y reportes de casos. Modelo de efecto ajustado RR e IC 95\%. Análisis heterogeneidad para resultados. Prueba de $\mathrm{I}^{2}$ heterogeneidad estadística. Riesgo de sesgo con riesgo Cochrane y ROBINS-1. Análisis de sesgos de reporte con la gráfica de embudo (funnel plots). Resultados: Se obtuvieron

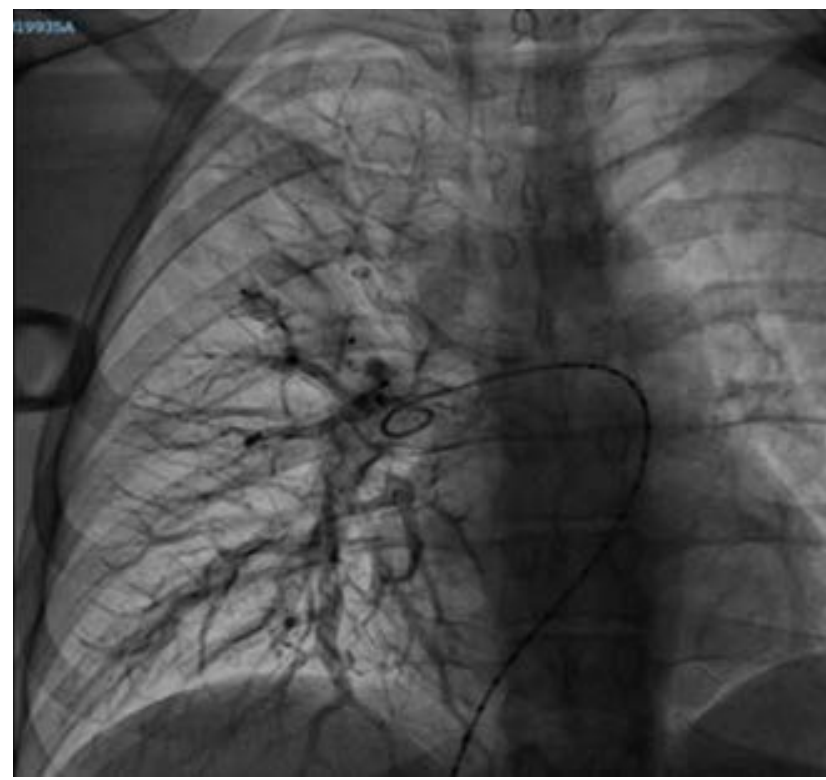

Figura 11.2.2.

Tabla 11.3.1: Comparación de incidencia de objetivos de seguridad en toda la cohorte y en los estudios incluidos en metaanálisis.

\begin{tabular}{|c|c|c|c|c|c|}
\hline Desenlace & $\begin{array}{l}\text { Alteplasa } 50 \mathrm{mg} \\
(\mathrm{n}=1,066) \\
\mathrm{n}(\%)\end{array}$ & $\begin{array}{l}\text { Alteplasa } 50 \text { mg } \\
\text { (metaanálisis) } \\
(\mathrm{n}=636) \\
\mathrm{n}(\%)\end{array}$ & $\begin{array}{l}\text { Alteplasa } 100 \mathrm{mg} \\
(\mathrm{n}=3,145) \\
\mathrm{n}(\%)\end{array}$ & $\begin{array}{l}\text { Alteplasa } 100 \text { mg } \\
\text { (metaanálisis) } \\
\text { (n= 624) } \\
n(\%)\end{array}$ & $\begin{array}{c}\text { Anticoagulación } \\
(\mathrm{n}=60) \\
\mathrm{n}(\%)\end{array}$ \\
\hline Mortalidad & 87/1,066 (8.1) & 72/636 (11.3) & 659/3,145 (20.9) & 86/624 (13.7) & $3 / 60(5.0)$ \\
\hline Hemorragia mayor & 28/1,066 (2.6) & 23/636 (3.6) & $151 / 3,145(4.8)$ & $31 / 624(4.9)$ & $0 / 60(0)$ \\
\hline Hemorragia intracraneal & $5 / 1,066(0.4)$ & $3 / 636(0.4)$ & $16 / 3,145(0.5)$ & $4 / 624(0.6)$ & $0 / 60(0)$ \\
\hline Hemorragia menor & $64 / 1,066(6.0)$ & $51 / 636(8.0)$ & $199 / 3,145$ (6.3) & $40 / 624(6.4)$ & $0 / 60(0)$ \\
\hline Recurrencia de TEP & $43 / 1,043 *(4.1)$ & $36 / 613 *(5.8)$ & $254 / 3,122 *(8.1)$ & $42 / 601 *(6.9)$ & $3 / 60(5.0)$ \\
\hline Tratamiento escalado & $381 / 757 *(50.3)$ & $295 / 548 *(53.8)$ & $1,448 / 3,069 *(47.1)$ & $227 / 548 *(41.4)$ & $* * *$ \\
\hline Desarrollo de HPCTE & 9/255*(3.5) & $* *$ & $* *$ & $* *$ & $32 / 60(53.3)$ \\
\hline
\end{tabular}

* Totales de pacientes ajustados con base en estudios que analizaron este objetivo.

** Desarrollo de hipertensión pulmonar crónica embolica (HPCTE) no fue valorado en ambos grupos de metaanálisis y en 100 mg total.

*** Tratamiento escalado no fue valorado en grupo de anticoagulación. 
Estudio

\begin{tabular}{|c|c|c|c|c|c|c|}
\hline \multicolumn{2}{|c|}{ Alteplasa 50 mg } & \multicolumn{2}{|c|}{ Alteplasa 100 mg } & \multirow{2}{*}{$\begin{array}{c}\text { Peso } \\
(\%)\end{array}$} & \multirow{2}{*}{$\begin{array}{l}\text { Riesgo relativo, } \\
\text { IC } 95 \%\end{array}$} & \multirow[b]{2}{*}{ Riesgo relativo, IC 95\% } \\
\hline Eventos & Total & Eventos & Total & & & \\
\hline
\end{tabular}

Wang

Wang

Hasanoglu

Subtotal (IC 95\%)

Eventos totales

\begin{tabular}{|r|r|r|r|}
\hline 1 & 65 & 3 & \\
\hline 0 & 23 & 0 & \\
\hline 71 & 548 & 83 & 5 \\
\hline & 636 & & 624 \\
\hline 72 & & 86 & \\
\hline
\end{tabular}

\begin{tabular}{r|r}
\hline 53 & \\
23 & \\
548 & \\
624 & \\
\hline
\end{tabular}

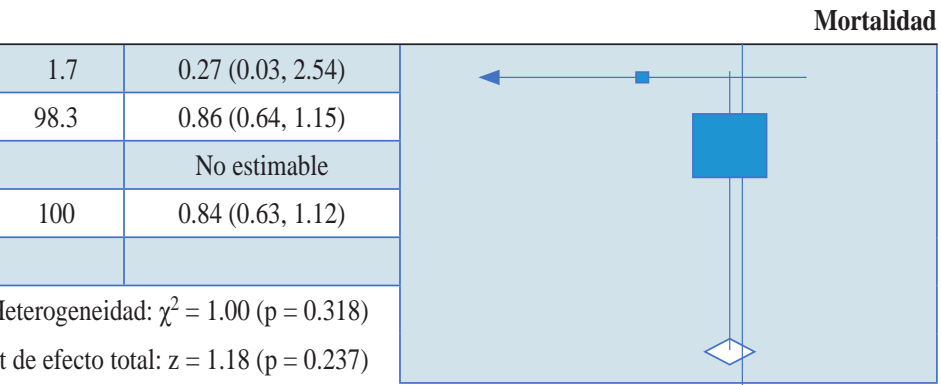

Test de efecto total: $\mathrm{z}=1.18(\mathrm{p}=0.237)$

Sangrado mayor

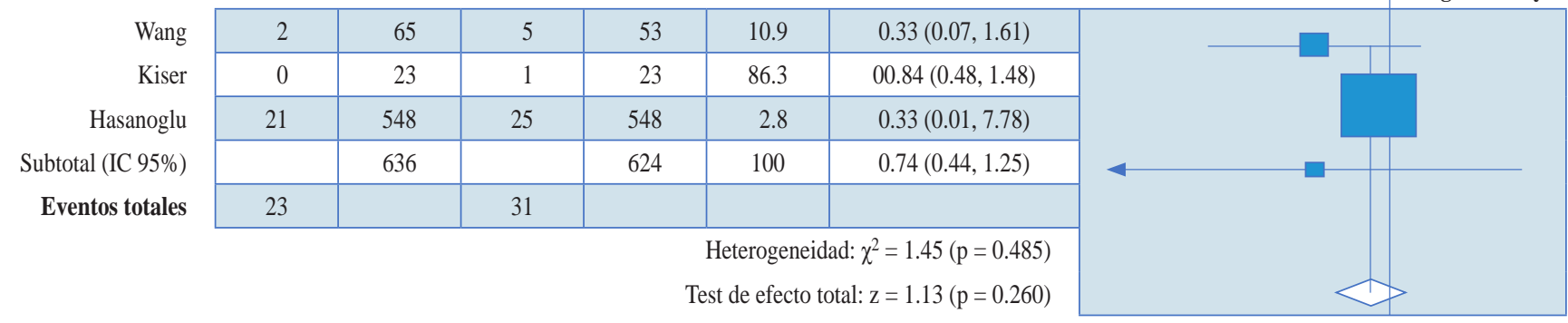

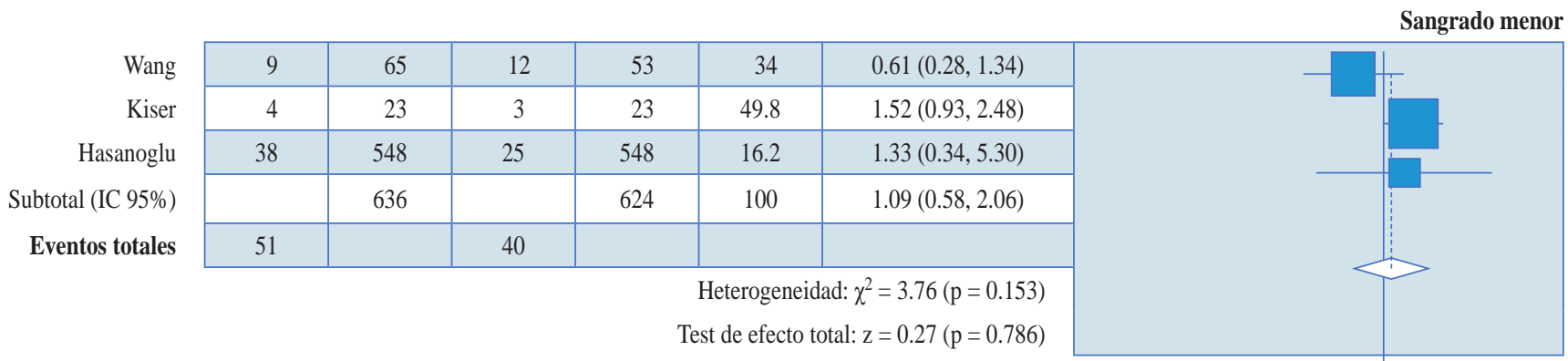

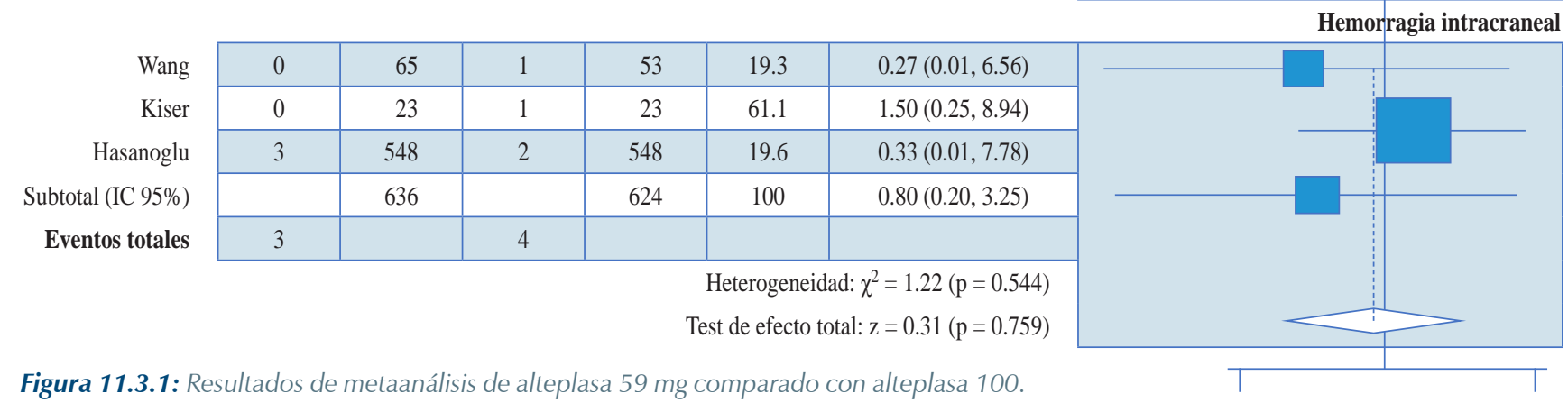

14,678 referencias, se incluyeron 17 para análisis cualitativo, tres para cuantitativo. Exclusión 14,629 referencias por el título y síntesis; 1,066 pacientes recibieron alteplasa $50 \mathrm{mg} / 1$-2 horas, 3145 alteplasa $100 \mathrm{mg} / 2$ horas y 60 sólo heparina. Objetivos de seguridad: menor tendencia de mortalidad con $50 \mathrm{mg}$ dosis versus 100 mg. Mayor mortalidad con anticoagulación. Similar comportamiento con hemorragias mayores, HIC y recurrencia. Metaanálisis: $50 \mathrm{mg}$ con tendencia no significativa para menor mortalidad versus $100 \mathrm{mg}$ (RR0.84; IC 95\% 0.63-1.12). Menor tendencia no significativa para hemorragias (RR 0.74; IC 95\%
0.44-1.25) con 50 versus 100 mg. Discusión: En TEP masiva y submasiva, $50 \mathrm{mg}$ alteplasa mejora el perfil de seguridad y mantiene la efectividad comparado con $100 \mathrm{mg}$ por tendencia a menor mortalidad, complicaciones hemorrágicas mayores y recurrencia.

\subsection{Trombólisis dirigida por catéter $y$ asistida por ultrasonido en $\geq 75$ años con tromboembolia pulmonar}


Castillo Pérez Mauricio, Jerjes Sánchez Carlos,

Rodríguez David, Paredes Vázquez José Gildardo, Panneflek Jathniel, Vázquez Guajardo Mauricio

Tecnológico de Monterrey.

Tipo de estudio: Reporte de casos clínicos con revisión de fuentes

Introducción: La trombólisis dirigida por catéter y asistida por ultrasonido (TDCAU) surge como opción terapéutica para mejorar la seguridad de la trombólisis en la tromboembolia pulmonar (TEP); sin embargo, en $\geq 75$ años su efectividad y seguridad es desconocida. Recientemente, en un octogenario con TEP submasiva Ilevado a TDCAU con efectividad, tuvimos lesión renal aguda (LRA) y lesión hepática isquémica. Descripción del caso: Considerando la falta de evidencia y recomendaciones, realizamos una revisión sistemática para identificar eventos adversos y evolución hospitalaria en $\geq 75$ años con TEP masiva y submasiva tratados con TDCAU. Metodología: Identificamos artículos publicados entre el 2008 y 2019 en las plataformas PubMed, Scopus, Web of Science y OpenGrey relacionados con $\geq 75$ años y TEP tratados con TDCAU. Se siguieron las guías PRISMA y fue registrada en PROSPERO. Además, realizamos otra revisión sistemática (pero sin restricción de edad) para actualizar la última revisión de TDCAU y poder comparar ambas poblaciones. Resultados: Identificamos 18 pacientes $\geq 75$ años (79.2, 75-86), en su mayoría del sexo femenino con TEP submasiva, así como dos eventos adversos relacionados al procedimiento con mortalidad (hemorragia intracraneal y ruptura de arteria pulmonar derecha). Identificamos dos casos de hemorragia mayor con lesión renal aguda, y un caso de lesión renal y hepática isquémica. De los pacientes que sobrevivieron, todos tuvieron mejoría clínica y ecocardiográfica. Al comparar este grupo con población más joven, observamos mayor tendencia a complicaciones hemorrágicas relacionadas con la edad (> 60 años) y tiempos de infusión prolongados. Discusión: Los resultados no sugieren riesgo prohibitivo de la TDCAU en $\geq 75$ años con TEP submasiva y masiva. Considerando el estudio OPTALYSE, es posible reducir complicaciones al reducir tiempos de infusión. 